\title{
Escala de Machismo Sexual: Evidências Psicométricas em Contexto Brasileiro
}

\author{
Francicléia Lopes Silva (iD \\ Universidade Federal da Paraíba, João Pessoa-PB, Brasil \\ Larisse Helena Gomes Macêdo Barbosa ${ }^{1}$ (D) \\ Universidade Cruzeiro do Sul, São Paulo-SP, Brasil \\ Valdiney Veloso Gouveia (D) \\ Universidade Federal da Paraíba, João Pessoa-PB, Brasil \\ Débora Najda de Medeiros Viana (iD \\ Universidade Integradas de Patos, Patos-PB, Brasil \\ Ana Karolyne Florencio Amorim (D) \\ Universidade Federal da Paraíba, João Pessoa-PB, Brasil
}

\section{RESUMO}

O presente artigo objetivou adaptar a Escala de Machismo Sexual para o contexto brasileiro, reunindo evidências de seus parâmetros psicométricos. Contou-se com uma amostra não probabilística de 219 universitários (Estudo 1) e 200 indivíduos (Estudo 2), com médias de idade semelhantes $(M=21,6 ; D P=4,06 ; M=19,0, D P=5,20$; respectivamente), sendo distribuídos igualmente em relação ao sexo no Estudo 1 e a maioria do sexo feminino no Estudo 2 (68,0\%). Estes responderam a Escala de Machismo Sexual, a Escala de Sexismo Ambivalente e perguntas demográficas. Os estudos revelaram uma solução unifatorial, com indicadores de consistência interna satisfatórios $(\alpha=0,81$ e $\alpha=0,76)$ e validade convergente confirmada por meio da correlação com o fator Sexismo Hostil e Sexismo Benévolo. Ademais, uma análise fatorial confirmatória corroborou tal dimensão preconizada. Conclui-se que essa medida se mostrou psicometricamente adequada para utilização no referido contexto.

Palavras-chave: machismo; validade; consistência interna.

\section{ABSTRACT - Sexual Machismo Scale: Psychometric Evidence in the Brazilian Context}

The current paper sought to adapt the Sexual Machismo Scale to the Brazilian context, gathering evidence of its psychometric parameters. Non-probabilistic samples of 235 undergraduate students (Study 1) and 200 individuals (Study 2), with similar mean ages $(M=21.6 ; S D=4.06 ; M=19.0, S D=5.20$; respectively), equally distributed by the sex in Study 1 and mostly female in Study $2(68.0 \%)$ were used. Participants completed the Sexual Machismo Scale, the Ambivalent Sexism Scale and demographic questions. The studies showed a single-factor solution, presenting suitable indices of internal consistency $(\alpha=0.81$ and $\alpha=0.76)$ and confirming the convergent validity through correlations with the hostile and benevolent sexism factors of the Ambivalent Sexism Scale. Additionally, a confirmatory factor analysis corroborated the previous solution. It was concluded that this measure seems to be psychometrically suitable for use in the context mentioned.

Keywords: machismo; validity; internal consistency.

\section{RESUMEN - Escala Machismo Sexual: Evidencias Psicométricas en el Contexto Brasileño}

El presente artículo objetivó adaptar la Escala de Machismo Sexual para el contexto brasileño, reuniendo evidencias de sus parámetros psicométricos. La muestra no probabilística estuvo compuesta por 219 universitarios (Estudio 1) y 200 individuos (Estudio 2) de la población general, con promedios de edad similares $(\underline{X}=21,6 ; D S=4,06 ; \underline{X}=19,0 ; D S=5,20$, respectivamente), siendo distribuidos igualmente en relación con el sexo en el Estudio 1, por otro lado, el Estudio 2 contó con mayoría del sexo femenino (69,9\%). Los participantes respondieron a la Escala de Machismo Sexual, la Escala de Sexismo Ambivalente y preguntas demográficas. Los estudios revelaron una solución unifactorial, con indicadores de consistencia interna satisfactorios $(\alpha=0,81$ y $\alpha=0,76)$ y validez convergente confirmada por medio de la correlación con el factor Sexismo Hostil y Sexismo Benévolo de la Escala de Sexismo Ambivalente. Además, un análisis factorial confirmatorio corroboró con los resultados preconizados. Se concluye que esta medida se mostró psicológicamente adecuada para su uso en dicho contexto.

Palabras clave: machismo; validez; consistencia interna.

O machismo se constitui na crença de superioridade do homem sobre a mulher, que deriva de condutas sexuais que põem em risco a saúde sexual e o próprio bem-estar físico de si e de seus parceiros e parceiras (Rodríguez,

Endereço para correspondência: Rua Radialista Antonia Assunção de Jesus, Bancários, 58052-230, João Pessoa, PB. Tel.: (83) 99699-9322.

E-mail: larissehelena@hotmail.com

Artigo derivado da 'Tese de doutorado' de 'Larisse Helena Gomes Macêdo Barbosa' com orientação de 'Valdiney Veloso Gouveia, defendida em '2017 no programa de pós-graduação 'Programa de Pós-Graduação em Psicologia Social' da 'Universidade Federal da Paraíba'. 
Rodríguez, \& Ramirez 2010). Fomentando bases hierárquicas entre os gêneros, o machismo está implícito em instituições culturais (e.g., a família, a heterossexualidade obrigatória), a qual transforma o masculino e o feminino biológicos em gêneros distintos e hierárquicos, sendo estas empregadas por leis que estruturam e incitam o desenvolvimento psíquico individual (Butler, 2003).

Nesse sentido, o machismo oferece modelos de identidade tanto para unidades masculinas como femininas, sendo amplamente aceitos por todos e avaliados por liderança ou hegemonia masculina (Drumont, 1980). Essa categorização de papéis de gênero oferecida pelo machismo deriva em violências de gênero, principalmente contra as mulheres. Segundo Peixoto e Nobre (2015), o machismo por si só já se configura em uma forma de violência contra a mulher, uma vez que fomenta uma cultura de distinção extrema de direitos e deveres entre os gêneros, levando ao preconceito e a violências mais evidentes.

Nessa relação de poder exercida pelo machismo, onde se constitui o masculino e o feminino, ele mesmo se encontra numa relação indissociável do sexismo (Rodríguez et al., 2010). Nessa perspectiva, Glick e Fiske (1996) o definem como um conjunto de estereótipos sobre a avaliação cognitiva, afetiva e atitudinal acerca de papéis pertinentes na sociedade, orientada aos indivíduos de acordo com o seu sexo, tendo suas raízes em condições biológicas, históricas e sociais.

Diferente de outras formas de intolerância social, como o racismo, por exemplo, o sexismo não é regido por uma única dimensão; o sexismo ambivalente apresenta duas formas principais: a hostil e a benevolente, sendo a primeira forma a expressão mais explícita do preconceito contra a mulher (Formiga, Gouveia, \& Santos, 2002). O sexismo benevolente é representado pela idealização da mulher como a figura protetora e respeitosa de "mãe", alocando-a na posição de objeto romântico. Segundo Glick e Fiske (1996), os sexismos hostil e benevolente estão interligados por três subcomponentes: paternalista, diferenciação de gênero e heterossexualidade. O paternalismo diz respeito à forma de um pai se relacionar com seu filho, caracterizada por dominação e proteção; a diferenciação de gênero surge a partir dos papéis sociais, sendo sustentada pela diferenciação do sexo biológico; e o componente da heterossexualidade parte do princípio de complementariedade, onde uma mulher não será feliz sem um homem e vice-versa.

O machismo e o sexismo em seus aspectos conceituais e práticas se configuram como sinônimos. Porém, o machismo sexual é um fator que surge no ceio do machismo e do sexismo hostil, voltando-se, segundo Rodríguez, Rodríguez e Ramirez (2010), a condutas, atitudes e crenças que circundam o sexual, colocando em xeque as relações de uma sexualidade desregrada e de risco para a saúde dos indivíduos envolvidos. Neste artigo o interesse é com a dimensão do machismo, procurando avaliá-lo, razão que demanda conhecer as opções disponíveis.
Diversos instrumentos foram desenvolvidos para avaliar o machismo, hipermasculinidade e construtos relacionados no cenário internacional, contudo ele não é verificado quanto ao contexto nacional, o que motivou a realização do presente estudo, focado em adaptar uma medida a respeito. Todavia, antes de considerá-la parece pertinente ter em conta as medidas que foram propostas no âmbito internacional.

\section{Escalas de Machismo}

Alguns autores empreenderam esforços para construir instrumentos de medida do machismo. Por exemplo, Villemez e Toughey (1977) desenvolveram a "Macho" Scale, composta por 28 itens que enfocam estereótipos, discriminação e autoritarismo (e,g," "A esposa não deve contradizer o marido em público"; "É certo que uma mulher trabalhe, mas o pão permanece primariamente uma responsabilidade masculina"). Esses autores relataram confiabilidade teste-reteste para a escala, variando de 0,89 a 0,91 . Entretanto, não foram encontradas informações acerca dos índices de consistência interna de cada fator individualmente (Neff, 2001).

Mosher e Sirkin (1984) desenvolveram o Hypermasculinity Inventory (HMI) com o fim de medir a personalidade machista. Esse instrumento se compõe de 30 itens de escolha forçada, distribuídos em três fatores: Atitudes sexuais calosas em relação às mulheres (e.g., "Homem que é homem precisa fazer sexo regularmente"; "Homem que é homem pode ficar sem fazer sexo"), Violência como virilidade (e.g., "É natural que os homens entrem em brigas"; "A violência física nunca resolve um problema") e Perigo como excitante (e.g., "Eu gosto de festas selvagens e desinibidas"; "Eu gosto de festas tranquilas com boas conversas"). De acordo com esses autores, o alfa de Cronbach para o fator geral foi de 0,80 , estando próximo a 0,70 para os fatores específicos. Sua principal limitação, segundo Burk, Burkhart e Sikorski (2004), é quanto a seus aspectos conceituais, metodológicos e lexicais.

Partindo da HMI, Mosher (1991) desenvolveu a Expanded Hypermasculinity Index (EHMI). O EHMI agregou um conceito aos existentes, isto é, a dureza, de tal forma que as dimensões dessa medida incluem a violência como necessária, o sexo como direito, o perigo como excitante e a dureza como autocontrole. Essa medida é composta de 40 itens, apresentando alfa de Cronbach 0,94 para o fator geral, tendo este variado de 0,70 a 0,90 para seus fatores específicos. Os estudos com essa medida foram conduzidos majoritariamente com estudantes universitários, não sendo encontrados dados com a população geral (Neff, 2001).

Strong, McQuillen e Hughey (1993) propuseram o Macho Belief Inventor, elaborado a partir de uma perspectiva patológica do machismo. Essa medida é composta por 46 itens respondidos em escala de quatro pontos, tipo Likert. A análise fatorial mostrou uma estrutura de onze 
fatores, a exemplo de promiscuidade, experiências sexuais antes do casamento, dominação e supremacia masculina. A consistência interna dessa medida, considerando um fator geral, foi de 0,84. Apesar de se indicarem múltiplos fatores, não foram encontradas informações acerca da consistência interna de cada fator individualmente.

A Escala de Machismo (Machismo Scale) foi desenvolvida por Cuellar, Arnold e Gonzalez (1995), estando formada por 17 itens, que precisariam abordar tanto dimensões negativas (masculinidade, domínio sobre a família e a esposa) como positivas (masculinidade, bravura, força, independência, poder e proteção da família) do machismo. Ela apresenta quatro fatores: superioridade masculina (e.g., "Homens são mais inteligentes que as mulheres"; "Na maioria das vezes, é melhor ser homem do que mulher"), papel do gênero masculino (e.g., "Os meninos não devem brincar com bonecas e brinquedos de outras meninas"), papel do gênero feminino (e.g., "Existem alguns trabalhos que as mulheres simplesmente não deveriam ter"; "É mais importante para uma mulher aprender a cuidar da casa e da família do que para ela ter uma educação universitária") e força masculina (e.g., "A maioria das mulheres tem pouco respeito pelos homens fracos"; "É importante que um homem seja forte"). A consistência interna para a escala total foi de 0,78 . Sua principal limitação, segundo Neff (2001), é que essa medida foi desenvolvida em população de estudantes universitários estadunidenses.

A Machismo Scale (MS) foi desenvolvida por Arciniega, Anderson, Tovar-Blank e Tracey (2008) em população mexicana, estando formada por 20 itens que procuram representar os aspectos negativo e positivo do machismo, sendo respondidos em escala de sete pontos, tipo Likert, variando de 1 (Discordo totalmente) a 7 (Corcordo totalmente). Tais itens são distribuídos equitativamente em dois fatores: machismo tradicional (e.g., "Os homens são superiores às mulheres"; "Um homem deve estar no controle de sua esposa") e cavalherismo (e.g., "Os homens devem estar dispostos a lutar para defender sua família"; "Os homens devem mostrar boas maneiras em público"). Relataram-se alfas de Cronbach de 0,84 e 0,71 para os seus fatores machismo tradicional e cavalherismo, respectivamente. Umas de suas limitações foi ter sido desenvolvida e validada unicamente para pessoas do sexo masculino.

Scale M. (Cortada, Bertoni, \& Adamovsky, 1970) é composta por 22 itens, distribuídos em seis dimensões: imagem física (e.g., "A educação física é tão importante para o homem quanto a intelectual"," O homem é feito a força de golpes"), relações familiares (e.g., "Crianças do sexo masculino devem ser ensinadas a não orar"; "O marido é o único que governa em casa"), relações sexuais (e.g., "No homem, a infidelidade é perdoável"; "Em todas as circunstâncias, o homem deve afirmar sua virilidade"), autoimagem (e.g., "As mulheres são geralmente mais sensíveis que os homens"; "As mulheres são mais impulsivas que os homens") esfera de trabalho (e.g., "É degradante para os homens fazer trabalhos domésticos"; "Em algumas circunstâncias, a mulher deve assumir o controle do lar") e posição social relativa de homens e mulheres (e.g., "A discriminação entre os sexos é contraditória à igualdade humana", "Em todas as circunstâncias da vida, homens e mulheres são iguais"). Esse instrumento trata-se de uma escala dicotômica com opção de resposta 1 "concordo" e 0 "discordo", apresentanso consistência interna de 0,64. Apesar de indicar múltiplos fatores, não foram encontradas informações acerca da consistência interna de cada fator de forma individual.

Differential masculinity inventory (ADMI-60) foi desenvolvido por Burk et al. (2004). Ele é composto por 44 itens e consta com uma escala de resposta de 5 pontos, variando de 1 (concordo totalmente) a 5 (discordo totalmente). Esse instrumento é representado em cinco fatores: hipermasculinidade (e.g., "Considero homens superiores às mulheres no intelecto"; "Eu valorizo o poder sobre outras pessoas"), identidade sexual (e.g., "Eu acho que é bom para garotos adolescentes fazerem sexo"; "Não me sinto culpado quando traio minha namorada/esposa"), dominância e agressão (e.g., "Não me importo de usar violência física para defender o que tenho"; "Muitos homens não são tão durões quanto eu”), masculinidade conservadora (e.g., "Eu me considero bastante superior à maioria dos outros homens"; "Eu gosto de ser o chefe") e desvalorização das emoções (e.g., "Eu acho que homens que choram são fracos"; "Eu tento evitar conflitos físicos"), apresentando consistência interna variando de 0,85 , a 0,73 . Os próprios autores apontam para uma limitação do instrumento quanto a interpretação dos itens dos fatores 3 e 4 que se fazem complexos, além do instrumento ter sido desenvolvido unicamente para a população masculina.

Estudos têm utilizado o construto para avaliar o machismo de estudantes de medicina peruanos (Mejia, Pulido-Flores, Quiñones-Laveriano, Nieto-Gutierrez, \& Heredia, 2018), comparar condutas de autoeficácia e o machismo sexual entre gerações (Díaz-Rodríguez \& González-Ramírez, 2012), medir a percepção e atitudes frente gênero e violência de gênero (Otero \& Coronado, 2019), avaliar o machismo sexual de pais e sua relação com a prevenção do vírus papiloma humano em adolescentes (Dávila, 2017) e medir a satisfação sexual e apego adulto em mulheres (Barreto \& Coico, 2017). A Escala de Machismo Sexual também é recomendada para avaliar o machismo nos homens a fim de intervir socialmente para reeducá-los no que tange às novas possibilidades de uma masculinidade que seja pautada na igualdade dos gêneros (Avilés \& Vigo, 2019). Em suma, a Escala de Machismo Sexual tem demonstrado ser um instrumento útil que facilita estudos transculturais, oportuniza considerar outros construtos e fornece uma medição fidedigna do machismo para 
fins de intervenção social. Além disso, garante menor estresse do participante no momento de responder devido o menor número de itens (12 itens).

Reconhecendo que as escalas para avaliar o machismo têm incluído um número grande de itens, Rodríguez et al. (2010) desenvolveram mais recentemente uma medida abreviada e com ênfase na sexualidade, denominada como Escala de Machismo Sexual, cujo propósito é avaliar comportamentos, atitudes e crenças machistas do ponto de vista sexual em homens e mulheres. Esse tipo de medida facilita estudos transculturais, oportuniza considerar outros construtos no estudo e garantem menor estresse do participante no momento de responder devido o menor número de itens (12 itens). Reconhecendo a qualidade de seus parâmetros psicométricos e a vantagem de ser uma medida curta e objetiva, inexistindo instrumentos dessa natureza adequado à realidade brasileira, decidiu-se adaptá-la. Procura-se, portanto, descrevê-la com maior detalhe a seguir.

A Escala de Machismo Sexual foi elaborada pensando no contexto hispânico. Seus autores pretenderam contar com um instrumento breve, válido e confiável, capaz de avaliar comportamentos, atitudes e crenças machistas desde a perspectiva sexual. Nesse sentido, essa escala considerou participantes do México, onde o machismo é parte da cultura, constituída por itens elaborados com linguagem adequada e genérica em termos de idade, sexo e preferência sexual. Essa medida foi inicialmente composta por 24 itens, sendo posteriormente reduzida a 12 itens (e.g., "Somente homens podem fazer sexo antes do casamento"; "É dever da mulher se cuidar na relação sexual") que, após as análises fatoriais exploratória e confirmatória, foram agrupados em estrutura unifatorial cujo alfa de Cronbach foi de 0,91. Tais itens foram respondidos em escala de 5 pontos, tipo Likert, variando de 1 (Discordo totalmente) a 5 (Concordo totalmente) (Díaz-Rodríguez \& González-Ramírez, 2012).

Considerando os instrumentos disponíveis na literatura, sobretudo não tendo sido encontrada uma medida psicometricamente adequada e adaptada ao contexto brasileiro, decidiu-se ter em conta a Escala de Machismo Sexual (Rodríguez et al., 2010), reunindo evidências de sua validade fatorial, validade de critério e consistência interna. Nessa direção, realizaram-se os dois estudos a seguir descritos.

\section{Estudo 1. Adaptação da Escala de Machismo Sexual (SEM-Sexismo-12)}

Tratou-se da primeira tentativa de adaptar essa medida para o contexto brasileiro. Essencialmente, checaram-se sua validade fatorial e consistência interna, empregando procedimentos exploratórios. Definida sua estrutura, haveria possibilidade de testá-la em um estudo independente, reforçando a adequação psicométrica dessa escala.

\section{Participantes}

\section{Método}

Participaram desse estudo 235 estudantes universitários de Patos (Paraíba) com idade média de 21,7 anos $(D P=4,06$, variando de 17 a 39 anos), distribuídos quase igualmente em relação ao sexo (50,4\% masculino), sendo a maioria solteira $(87,8 \%)$. Tratou-se de amostra de conveniência (não probabilística), tendo participado as pessoas que, presentes em sala de aula e convidadas a colaborar, concordaram em fazê-lo voluntariamente.

\section{Instrumentos}

Os participantes responderam um questionário demográfico (sexo, idade, escolaridade, religião, religiosidade e estado civil), disposto ao final, e a Escala de Machismo Sexual (SEM-Sexismo-12) (Rodríguez et al, 2010). Como anteriormente se indicou, essa escala se constitui de 12 itens respondidos em escala de 5 pontos, tipo Likert, variando de 1 (Discordo totalmente) a 5 (Concordo totalmente). Sua tradução foi feita a partir da versão em espanhol por uma pesquisadora bilíngue, posteriormente retraduzida para o idioma original por outra pesquisadora bilíngue. Um pesquisador também bilíngue comparou as duas versões em espanhol, observando a manutenção dos conteúdos abordados pelos itens, dando por adequada a versão em português dessa medida. Como etapa prévia à sua aplicação, decidiu-se proceder à sua validação semântica, considerando a colaboração de cinco estudantes de uma universidade privada. Verificou-se, então, a compreensão das instruções sobre como respondê-la, do conteúdo de seus itens e da escala de resposta adotada. Esse procedimento não demandou quaisquer alterações substanciais, podendo ser solicitada essa versão a um dos autores.

\section{Procedimento}

Inicialmente, o projeto foi submetido ao Comitê de Ética, tendo sido aprovado, após a emissão do parecer de número 1.877.329. Posteriormente, contataram-se os potenciais participantes na praça de alimentação da faculdade, passando a abordá-los com o fim de que colaborassem respondendo os questionários impressos, assegurando-lhes o anonimato e a confidencialidade de suas respostas. Todos assinaram o Termo de Consentimento Livre e Esclarecido, conforme define a Resolução $n^{\circ}$ 512/16, do Conselho Nacional de Saúde, demandando-se cerca de 10 minutos para concluir sua participação no estudo.

\section{Análises de Dados}

Os dados foram analisados com o SPSS (versão 21). Calcularam-se estatísticas descritivas (e.g., média, desvio padrão, frequência), testes $t$ de Student (comprovação do poder discriminativo dos itens), análise dos eixos principais e análise paralela. Foi verificada, ainda, a consistência interna (alfa de Cronbach) da medida de machismo. 


\section{Resultados}

Inicialmente, checou-se o poder discriminativo dos itens a partir de grupos-critério interno, examinando se os itens diferenciavam os respondentes com magnitudes próximas no traço latente de interesse (machismo). No caso, adotou-se a mediana empírica da pontuação total média; definiram-se então os grupos-critério inferior e superior a partir da mediana $(M d=1,50)$, usando o teste $t$ de Student para amostras independentes a fim de comparar suas médias para cada item. Excetuando o item 3 (Só os homens podem ter experiência sexual) que não diferenciou os grupos, todos os demais o fizeram. Desse modo, decidiu-se mantê-lo em análises subsequentes para observar como se comportaria.

No que se refere à fatorabilidade da matriz de correlação inter-itens, consideraram-se como indicadores que atestaram sua adequação: o teste de adequação da amostra de Kaiser-Meyer-Olkin (KMO), cujo resultado foi de 0,80 , e o teste de esfericidade de Bartlett, que apresentou um resultado também satisfatório $\left[\chi^{2}(66)=797,78, p<0,001\right]$. Portanto, ficou demonstrada a pertinência de ser realizar uma análise dos eixos principais com os dados. Desse modo, procedeu-se com essa análise sem fixar o tipo de rotação ou o número de componentes a extrair.

Quanto ao número de componentes a extrair, tiveram-se em conta múltiplos indicadores. Inicialmente, considerou-se o critério de Kaiser (valor próprio igual ou superior a 1), observou-se a possibilidade de extrair até quatro componentes $(4,10,1,65,1,31$ e 1,03), que explicaram conjuntamente $67,4 \%$ da variância total. Posteriormente, adotou-se o critério de Cattell (distribuição gráfica dos valores próprios, desprezando aqueles a partir da configuração do "cotovelo", i.e. ponto de inflexão da curva), que permitiu identificar até quatro componentes, embora o primeiro tenha ficado mais evidente, conforme a Figura 1. Por fim, checou-se a solução mais adequada por meio do critério de Horn (análise paralela, que avaliar a preponderância dos valores próprios observados em relação aos simulados). Nesse caso, adotaram-se os mesmos parâmetros do banco original de dados, contendo 235 participantes e 12 itens, realizando 1.000 simulações.

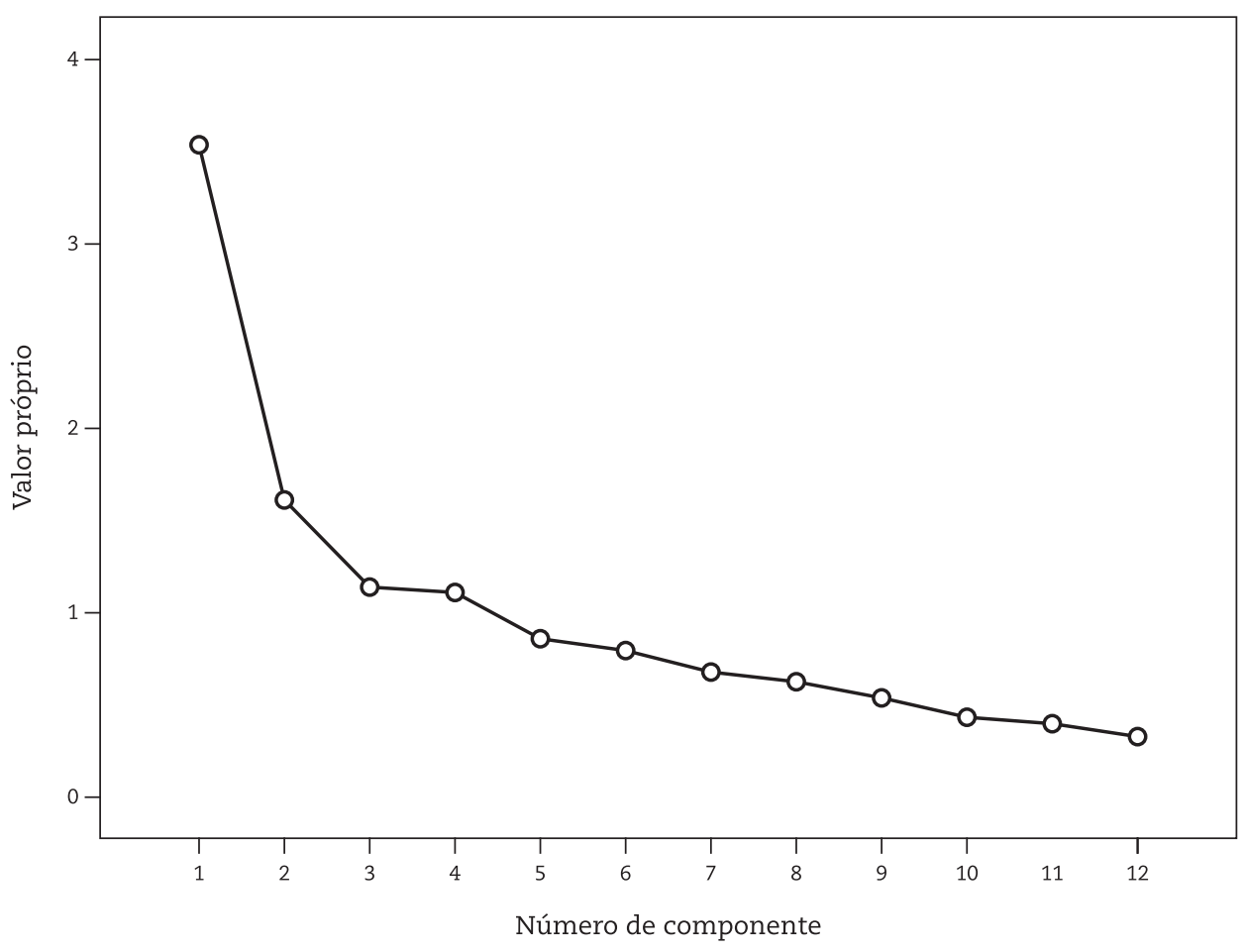

Figura 1. Distribuição Gráfica dos Valores Próprios

Os quatro primeiros valores próprios gerados aleatoriamente foram como seguem: 1,49, 1,37, 1,26 e 1,19. Comparando esses valores com aqueles descritos previamente, que foram observados empiricamente, sugere-se a possibilidade de reter até três componentes. Entretanto, com a finalidade de testar uma estrutura mais parcimoniosa, optou-se por extrair um componente, coerente com a estrutura originalmente relatada por Rodríguez et al. (2010). Portanto, fixou-se a extração de um único componente, admitindo-se saturação mínima do item de 
|0,30| (Hair, Black, Babin, \& Anderson, 2015), unicamente o item 4 (Um homem pode ter sua primeira relação sexual com uma pessoa que não seja sua companheira) não saturou acima do ponto de corte, no entanto, os alfas de Cronbach com $(\alpha=0,81)$ e sem $(\alpha=0,82)$ o item
4 não diferiram estatisticamente $(t<1)$. Dessa forma, intentando que o instrumento mantivesse a forma original, optou-se por não eliminá-lo; embora não desejável, há que se considerar que sua saturação $(0,24)$ difere estatisticamente de zero $(t=2,56, p<0,05)$.

Tabela 1

Estrutura Fatorial da Escala de Machismo Sexual

\begin{tabular}{clc}
\hline Itens & \multicolumn{1}{c}{ Descrição do conteúdo } & Saturação \\
\hline 07 & Um homem precisa ter várias parceiras sexuais. & 0,75 \\
06 & Uma mulher deve aceitar à infidelidade/traição do seu companheiro. & 0,70 \\
12 & O pai deve fazer com que seu filho inicie a vida sexual. & 0,69 \\
11 & O homem deve iniciar sua vida sexual na adolescência. & 0,64 \\
08 & Não importa a situação ou estado de humor da mulher, ela deve ter relações sexuais sempre & 0,60 \\
05 & Ume seu companheiro pedir. & 0,55 \\
10 & É dever da mulher se cuidar na relação sexual. & 0,49 \\
09 & Um homem pode ter várias parceiras sexuais ao mesmo tempo. \\
02 & Homens podem ter filhos fora do casamento & 0,45 \\
03 & Só os homens podem ter experiência sexual & 0,38 \\
01 & Somente homens podem fazer sexo antes do casamento. & 0,35 \\
04 & Um homem pode ter sua primeira relação sexual com uma pessoa que não seja sua companheira. & 0,24 \\
Número de itens & 0,33 \\
Valor próprio & 12 \\
Alfa de Cronbach & 4,10 \\
\hline
\end{tabular}

Como pode ser observado, a Escala de Machismo Sexual ficou formada por 12 itens que foram agrupados em um único componente, apresentando valor próprio de 4,10, responsável pela explicação de $28,9 \%$ da variância total. Tais itens apresentaram saturação média de 0,51 , variando de 0,24 (Item 4 . Um homem pode ter sua primeira relação sexual com uma pessoa que não seja sua companheira) a 0,75 (Item 7 . Um homem precisa ter várias parceiras sexuais). Esse componente apresentou consistência interna (alfa de Cronbach, $\alpha$ ) de 0,81 . Portanto, os resultados foram na direção dos observados por Rodríguez et al. (2010), ressaltando-se, entretanto, a natureza exploratória das análises realizadas nessa oportunidade, o que demandou pensar em um novo estudo para comprovar essa estrutura unidimensional, o que se leva a cabo a seguir.

\section{Estudo 2. Teste da Estrutura Fatorial da Escala de Machismo Sexual}

Esse segundo estudo procura testar a adequação da estrutura unifatorial da medida de machismo sexual, além de conhecer sua consistência interna e evidência de validade de critério, correlacionando suas pontuações com aqueles de sexismo ambivalente. No caso, considerou-se uma amostra independente, realizando análise fatorial confirmatória para verificar evidências de validade fatorial dessa medida.

\section{Método}

\section{Participantes}

Participaram da pesquisa 200 pessoas da população geral. Estes tinham média de idade de 19 anos $(D P=5,20)$, sendo a maioria do sexo feminino (68\%) e solteira (91\%). Quanto à escolaridade, $21 \%$ tinham o ensino médio completo, 47,5\% estavam cursando a universidade e $12,5 \%$ concluíram o ensino superior. Tratou-se de uma amostra de conveniência, tendo participado as pessoas que, tendo sido convidadas ou sabendo do estudo, quiseram participar voluntariamente.

\section{Instrumentos}

Os participantes responderam um questionário contendo os mesmos instrumentos do Estudo 1 e Escala de Sexismo Ambivalente (ASI). Esta foi desenvolvida 
originalmente por Glick e Fiske (1996), tendo sido adaptada ao contexto brasileiro por Formiga et al. (2002). É composta por 22 itens que avaliam dois fatores: sexismo hostil (SH; e.g., "Mulheres procuram poder controlando aos homens"; "Mulheres exageram problemas no trabalho") e sexismo benevolente (SB; "Homem não se sente completo sem o amor de uma mulher"; "Todo homem deve ter uma mulher a quem amar"). Esses itens são respondidos em escala de seis pontos, tipo Likert, variando de 0 (Discordo totalmente) a 5 (Concordo totalmente).

\section{Procedimento}

A coleta de dados foi realizada por meio de um questionário on-line, requerendo que o participante fosse maior de 18 anos, concordando com o Termo de Consentimento Livre e Esclarecido. Procurando recrutar os potenciais participantes, o link para ter acesso ao questionário foi compartilhado em redes sociais (e.g., Facebook, Instagram). Quando a pessoa o acessava, conhecia primeiramente os objetivos da pesquisa e os cuidados éticos seguidos, indicando que se tratava de participação voluntária, assegurando o e sigiloso das respostas e a possibilidade de desistir de participar sem qualquer ônus. Nessa direção, seguiram-se os procedimentos preconizados pela Resolução CNS 510/16 (Brasil, 2016), tendo sido o projeto previamente aprovado por Comitê de Ética (Parecer de número: 1.877.329). Em média, as pessoas levaram cerca de 15 minutos para concluir sua participação.

\section{Análise dos Dados}

Utilizou-se o programa estatístico $R$ ( $R$ Development Core Team, 2011; Raiche, Walls, Magis, Riopel, \& Blais, 2013) para realizar a análise fatorial confirmatória (AFC), comprovando a estrutura unidimensional da Escala de
Machismo Sexual. No caso, considerou-se o estimador de Quadrados Mínimos Ponderados (WLSMV). Os seguintes indicadores de ajuste foram considerados (Byrne, 2013; Hooper, Coughlan, \& Mullen, 2008; Meade, Johnson, \& Braddy, 2006; Tabachnick \& Fidell, 2013): razão qui-quadrado $\left(\chi^{2}\right) /$ graus de liberdade $(g l)$, admitindo-se como satisfatório valor entre 2 e 3 , mas sendo aceito até 5 como indicador de adequação do modelo teórico; GFI (Goodness-of-Fit Index) e AGFI (Adjusted Goodness-ofFit Index), cujos valores variam de 0 a 1 , aceitando-se como indicadores de ajuste do modelo os próximos ou superiores a 0,90; CFI (Comparative Fit Index) e TLI (Tucker-Lewis Index), cujos valores também variam de 0 a 1 , admitindo-se como representando um ajuste adequado aqueles iguais ou superiores a 0,90; e RMSEA (Root-Mean-Square Error of Approximation), cujo valor recomendado para aceitar o modelo é igual ou inferior a 0,05 , admitindo-se até 0,08 ou, no caso do limite superior, 0,10 .

\section{Resultados}

Tomando como referência a estrutura fatorial observada no Estudo 1, especificou-se que todos os 12 itens da medida de machismo saturassem em um fator geral. Esse modelo apresentou os seguintes indicadores de ajuste: $\chi^{2 /}$ $g l=1,08, \mathrm{GFI}=0,99, \mathrm{AGFI}=0,99, \mathrm{CFI}=0,97, \mathrm{TLI}=0,96$ e RMSEA $=0,02(\mathrm{IC} 90 \%=0,000-0,050)$. Destaca-se que todas as saturações ( $\lambda$, lambdas) foram superiores a 0,19 , com valor médio de 0,42 [amplitude de 0,19 (item 10. É dever da mulher se cuidar na relação sexual) a 0,67 (item 7. Um homem precisa ter várias parceiras sexuais)], sendo estatisticamente diferentes de zero $(\lambda \neq 0 ; z>1,96$, $p<0,05)$. O resumo dessa estrutura fatorial pode ser visto na Figura 2 a seguir, que indica haver evidência de validade fatorial dessa medida.

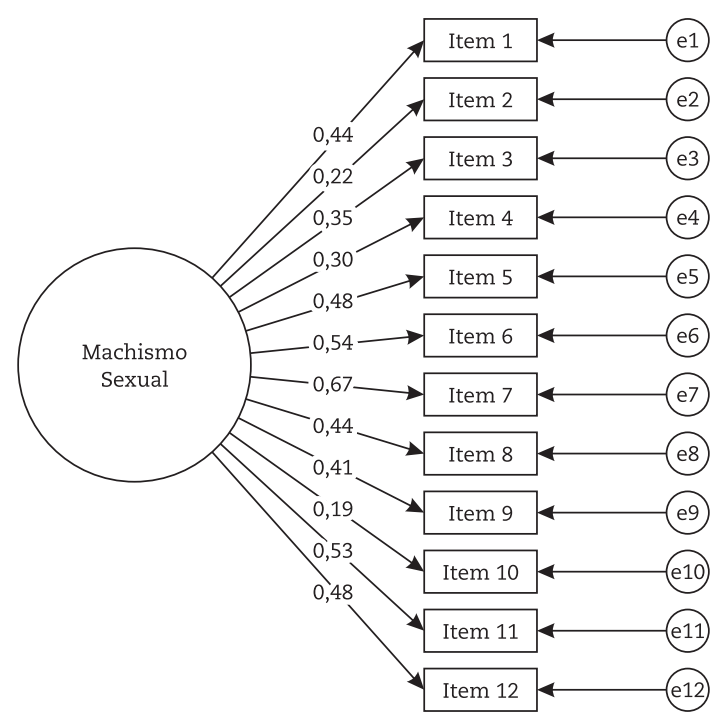

Figura 2. Estrutura Unifatorial da Escala de Machismo Sexual 
Esse fator geral apresentou consistência interna $(\alpha)$ de 0,65 , coeficiente que é estatisticamente significativo. Por fim, as pontuações nessa medida de machismo foram correlacionadas com aquelas dos fatores de sexismo ambivalente. Especificamente, observou-se que elas foram diretamente correlacionadas com o sexismo benevolente $(r=0,21, p<0,01)$ e, principalmente, o sexismo hostil $(r$ $=0,35, p<0,01)$, apoiando evidências de validade de critério. Frisa-se que a correlação do machismo sexual com esse tipo de sexismo foi mais forte do que com aquele $[z$ $(197)=2,56, p<0,05]$.

\section{Discussão}

As relações de gênero baseadas no machismo ainda estão presentes na sociedade contemporânea em atitudes e ações diversas. São relações construídas historicamente, que esboçam quais papéis sociais devem ser representados por homens e mulheres. Portanto, apesar das conquistas, as mulheres ainda se encontram em desvantagem em relação aos homens, pois são submetidas a situações de desigualdades variadas, como econômicas, sociais e sexuais (Silva \& Mendes, 2015). Precisamente em razão da relevância desse tema, decidiu-se empreender esforços para adaptar à realidade brasileira uma medida de machismo, lembrando, a Escala de Machismo Sexual (EMS) (Rodríguez et al., 2010).

A EMS, embora não seja a única que permite avaliar o construto de interesse, apresenta algumas vantagens em relação às demais medidas. Destacam-se os fatos de ela ter sido elaborada em contexto ibero-americano, compreendendo um instrumento breve e com itens simples, formulados de forma que não suscita ambiguidade, reunindo evidências de validade (fatorial e critério) e confiabilidade (consistência interna, alfa de Cronbach), sendo capaz de avaliar comportamentos, atitudes e crenças machistas desde uma perspectiva sexual, corroborando os achados quando de sua elaboração e comprovação inicial de seus parâmetros psicométricos (Rodríguez et al., 2010).

A propósito das evidências psicométricas da Escala de Machismo Sexual, os dois estudos realizados pareceram suportá-las, combinando estatísticas exploratórias e confirmatórias, procurando replicar os achados descritos por Rodríguez et al. (2010). No Estudo 1, embora fossem possíveis estruturas fatoriais mais complexas, a unifatorial se revelou adequada; apenas um item não funcionou adequadamente, tendo os demais apresentado saturações superiores ao que tem sido recomendado na literatura (0,30; Hair et al., 2015). Decidiu-se, ainda, checar a confiabilidade desta escala por meio do alfa de Cronbach, observando-se valores superiores ao ponto de corte comumente anunciado, inclusive para contexto de diagnóstico (0,70; Damásio, 2012).

Embora os achados do Estudo 1 tenham sido promissores, na linha do que se esperaria (Rodríguez et al.,
2010), reconheceu-se o caráter eminentemente exploratório das análises estatísticas realizadas. Desse modo, procurou-se realizar um novo estudo. Por meio de análise fatorial confirmatória, checou-se a possibilidade de tratar a EMS como unidimensional, observando-se indicadores de ajuste satisfatórios, que atenderam aos critérios mais exigentes da literatura (e.g., AGFI e CFI >0,90, RMSEA =0,05; Byrne, 2013; Tabachnick \& Fidell, 2013). A consistência interna dessa medida, como avaliada por meio do alfa de Cronbach, embora um pouco mais baixo do que no estudo anterior, não diferiu estatisticamente, estando acima do coeficiente geralmente recomendado (Pasquali, 2012).

Quanto às evidências de validade de critério no Estudo 2, as correlações das pontuações dessa medida com aquelas de sexismo foram na direção do esperado; por exemplo, segundo Rodríguez et al. (2010), o conteúdo dos itens da Escala de Machismo Sexual apresenta características do machismo tradicional, enfocando o papel mais submisso das mulheres, comparado com o dos homens, responsáveis pela tomada de decisão e controle do relacionamento, tendo participação ativa e determinante na sexualidade do casal. Nessa direção, coerentemente, foi mais forte a correlação das pontuações em machismo sexual com o sexismo hostil do que o benévolo. Isso reforça o que a literatura tem indicado, mostrando que o sexismo hostil é mais claro e diretamente discriminatório do que o benévolo (Formiga et al., 2002), ainda que esse último represente também uma forma de discriminação "branda" das mulheres (Glick \& Fiske, 1996).

Em resumo, o conjunto desses achados sugere evidências de validade (fatorial e critério) e consistência interna (alfa de Cronbach) da Escala de Machismo Sexual, o que apoia a adequação de seu uso no contexto de pesquisa. Não obstante, ainda restam ações a serem levadas a cabo em termos de direcionamentos futuros, a exemplo de conhecer evidências de sua estabilidade temporal (teste-resteste) e avaliar em que medida suas pontuações estão isentas do viés de resposta denominando como desejabilidade social (validade discriminante). No que diz respeito aos seus correlatos, será importante conhecer sua associação com construtos de natureza duradoura e universal, como os traços de personalidade e os valores humanos (Gouveia, 2013), que têm sido considerados como explicadores de diferenças individuais em temáticas relativas aos papéis sexuais (Guerra, Gouveia, Sousa, Lima, \& Freires, 2012). Talvez se possa pensar em um modelo hierárquico, concebendo os traços de personalidade como explicadores dos valores humanos e estes, por sua vez, explicando o machismo sexual e/ou as atitudes frente às vítimas de estupro.

Por fim, embora o objetivo principal deste artigo tenha sido alcançado, isto é, adaptar a EMS para o contexto brasileiro, reunindo evidências de sua adequação psicométrica, reconhecem-se limitações potenciais dos 
estudos. Por exemplo, consideraram-se amostras foram de conveniência, não sendo representativas da população brasileira ou mesmo da paraibana. Porém, há que frisar que não foi o propósito assegurar validade externa dos achados (generalização), mas reunir evidências a respeito dos parâmetros psicométricos da escala em pauta. Nesse caso, o número de participantes parece ter sido suficiente para as análises estatísticas realizadas (e.g., análises fatoriais exploratória e confirmatória, cálculo da consistência interna). Além disso, poder-se-ia ter considerado alguma medida alternativa de machismo, evitando admitir a legitimidade da EMS, o que favoreceriam evidências de validade convergente da escala ora tratada.

\section{Agradecimentos}

Não há menções.

\section{Financiamento}

A presente pesquisa não recebeu nenhuma fonte de financiamento sendo custeada com recursos dos próprios autores.

\section{Contribuições dos autores}

Declaramos que todos os autores participaram da elaboração do manuscrito. Especificamente, os autores Francicléia Lopes Silva e Ana Karolyne Florencio Amorim, participaram da redação inicial do estudo - conceitualização, investigação, visualização, a autora Larisse Helena Gomes Macêdo Barbosa participou da análise dos dados. Os autores Valdiney Veloso Gouveia e Débora Najda de Medeiros Viana participaram da redação final do trabalho - revisão e edição.

\section{Disponibilidade dos dados e materiais}

Todos os dados e sintaxes gerados e analisados durante esta pesquisa serão tratados com total sigilo devido às exigências do Comitê de Ética em Pesquisa em Seres Humanos. Porém, o conjunto de dados e sintaxes que apoiam as conclusões deste artigo estão disponíveis mediante razoável solicitação ao autor principal do estudo.

\section{Conflito de interesses}

Os autores declaram que não há conflitos de interesses.

\section{Referências}

Arciniega, G. M., Anderson, T. C., Tovar-Blank, Z. G., \& Tracey, T. J. (2008). Toward a fuller conception of Machismo: Development of a traditional Machismo and Caballerismo scale. Journal of Counseling Psychology, 55(1), 19-33. doi: 10.1037/0022-0167.55.1.19

Avilés, B. A. F., \& Vigo, E. A. (2019). Evaluación del impacto en el trabajo com los hombres desde la inteverción social. Em J. S. Albelda \& R. C. Paredes (Eds.). I Congreso internacional sobre masculinidades e igualdad: en busca de buenas prácticas de masculinidades igualitarias desde el ámbito de la Universidad (pp. 23-39). Universidad Miguel Hernández.

Barreto, S. J., \& Coico, G. L.H. (2017). Satisfacción sexual y apego adulto em mujeres estudiantes de una universidad privada [Tese de doutorado]. Universidade Señor de Sípan, Facultad de Humanidades, Escuela Académico Profesional de Psicología, Pimentel: Perú.

Brasil, Conselho Nacional de Saúde. (2016). Resolução CNS 510, de 07 de abril de 2016. Recuperado de http://conselho.saude.gov.br/ resolucoes/2016/Reso510.pdf

Butler, J. P. (2003). Proibição, psicanálise e a produção de matriz heterossexual. Em J. P. Butler. (pp. 61-109). Problemas de gênero: Feminismo e subversão da identidade. Rio de Janeiro: Civilização Brasileira.

Burk, L. R., Burkhart, B. R., \& Sikorski, J. F. (2004). Construction and preliminary validation of the auburn differential masculinity inventory. Psychology of Men E Masculinity, 5(1), 4-17. doi: 10.1037/1524-9220.5.1.4

Byrne, B. M. (2013). Structural equation modeling with Amos: Basic concepts, applications, and programming. London: Routledge.

Cortada, K. N,. Bertoni, A. L., \& Adamovsky, E. (1970). Un estudio experimental sobre el machismo. Revista Latinoamericana de Psicología, $2(1), 31-54$

Cuellar, I., Arnold, B., \& González, G. (1995). Cognitive referents of acculturation: Assessment of cultural constructs in Mexican Americans. Journal of Community Psychology, 23(4), 339-356. doi: 10.1002/1520-6629(199510)23:4<339::AID-JCOP2290230406>3.0.CO;2-7

Damásio, B. F. (2012). Uso da análise fatorial exploratória em psicologia. Avaliação Psicológica, 11(2), 213-228.

Dávila, S. P. E. (2017). Rol de los padres para la prevención del vírus del papiloma humano en adolescentes [Tese de doutorado]. Universidad Autónoma de Nuevo León. Facultad de Enfermería: México.

Díaz-Rodríguez, C. L., \& González-Ramírez, M. T. (2012). Comparación intergeneracional del machismo sexual y conductas de autoeficacia. Acta de Investigación Psicológica, 2(2), 638-649.

Drumont, M. P. (1980). Elementos para uma análise do machismo. Perspectivas, 3, 81-85.

Formiga, N. S., Gouveia, V. V., \& Santos, M. N. (2002). Inventário de sexismo ambivalente: sua adaptação e relação com o gênero. Psicologia em Estudo, 7(1), 103-111.

Glick, P., \& Fisk, S. T. (1996). The ambivalent sexism inventory: Differentiating hostile and benevolent sexism. The Amerycan Psychological Assiciation, 70(3), 491-512. doi: 10.1037/0022-3514.70.3.491

Gouveia, V. V. (2013). Teoria funcionalista dos valores humanos: Fundamentos, aplicações e perspectivas. São Paulo: Casa do Psicólogo.

Guerra, V. M., Gouveia, V. V., Sousa, D. M., Lima, T. J., \& Freires, L. A. (2012). Sexual liberalism-conservatism: The effect of human values, gender, and previous sexual experience. Archives of Sexual Behavior, 41(4), 1027-1039. doi: 10.1007/s10508-012-9936-4

Hair, J. F. J., Black, W. C., Babin, B. J., \& Anderson, R. E. (2015). Multivariate Data Analysis (7th ed.). Upper Saddle River, NJ: Prentice Hall.

Hooper, D., Coughlan, J., \& Mullen, M. (2008). Structural Equation Modelling: Guidelines for Determining Model Fit. Eletronic Journal of Business Research Methods. 6(1), 53-60. 
Meade, A. W., Johnson, E. I. C., \& Braddy, P. W. (2006). The utility of alternative fit indices in tests of measurement invariance. Em Academy of Management Best Conference (pp. B1-B6). Briarcliff Manor: New York.

Mejia, C. R., Pulido-Flores, J., Quiñones-Laveriano, D.M, Nieto-Gutierrez, W, \& Heredia, P. (2018). Machismo entre los estudiantes de medicina peruanos: Factores socio-educativos relacionados en 12 universidades peruanas. Revista Colombiana de Psiquiatria, 48(4), 215221. doi: $10.1016 /$ j.rcp.2018.02.001

Mosher, D. L. (1991). Macho men, machismo, and sexuality. Annual Review of Sex Research, 2(1), 199-247. doi: 10.1080/10532528. 1991.10559871

Mosher, D. L., \& Sirkin, M. (1984). Measuring a macho personality constellation. Journal of Research in Personality, 18(2), 150-163. doi: $10.1016 / 0092-6566(84) 90026-6$

Neff, J. A. (2001). A Confirmatory Factor Analysis of a Measure of "Machismo" among Anglo, African American, and Mexican American Male Drinkers. Hispanic Journal of Behavioral Sciences, 23(2), 171-188. doi: 10.1177/0739986301232004

Otero, L. M. R., \& Coronado, M. Z. (2019). Percepción y actitudes hacia el género y la violencia de género en alumnado universitario mexicano de Trabajo Social. Revista de Treball Social, 216, 105-123. doi: 10.32061/RTS2019.216.06

Pasquali, L. (2012). Análise fatorial para pesquisadores. Brasília, DF: LabPam.

Peixoto, A. F., \& Nobre, B. P. R. (2015). A responsabilização da mulher vítima de estupro. Revista Transgressões, ciências criminais em debate. $3(1), 227-239$.

Raiche, G., Theodore, A. W., David, M., Riopel, M., \& Blais, J. G. (2013). Methodology. 9(1), 23-29. doi: 10.1027/1614-2241/a000051

R Core Team. (2019). R: A language and environment for statistical computing. Vienna, Austria: R Foundation for Statistical Computing. Recuperado de https://www.Rproject.org/

Rodríguez, C. L. D., Rodríguez, M. A. R., \& Ramírez, M. T. G. (2010) Escala de machismo sexual (SEM-Sexismo-12): Diseño y análisis de propriedades psicométricas. SUMMA Psicológica UST, 7(2), 35-44.

Silva, M. C. \& Mendes, O. M. (2015). As marcas do machismo no cotidiano escolar. Caderno Espaço Feminino, 28(1), 90-99.

Strong, W. F., McQuillen, J. S, \& James D. Hughey, J. D. (1993). En el laberinto de machismo: A comparative analysis of macho attitudes among Hispanic and Anglo college students. Howard Journal of Communications, 5(1-2), 18-35. doi: 10.1080/10646179309361649

Tabachnick, B. G., \& Fidell, L. S. (2013). Using multivariate statistics. Boston, MA: Allyn and Bacon. 6.

Villemez, W. J., \& Touhey, J. C. (1977). A measure of individual differences in sex stereotyping and sex discrimination: The Macho scale. Psychological Reports, 41(2), 411-415. doi: 10.2466/pr0.1977.41.2.411

\section{Sobre os autores}

Francicléia Lopes Silva é psicóloga (UniFIP), mestra em psicologia social (UFPB), especialista em avaliação psicológica (UniFIP) e aluna de doutorado no Programa de Pós Graduação em Psicologia Social (UFPB).

Larisse Helena Gomes Macêdo Barbosa é professora da Uni FIP e Universidade Cruzeiro do SP. Psicóloga (UFPB), mestre e doutora em Psicologia Social (UFPB (UFPB).

Valdiney Veloso Gouveia é professor titular (UFPB), psicólogo (UFPB) e bacharel em direito (UNIPÊ), especialista em psicometria e mestre em psicologia social e do trabalho (UNB), doutor em psicologia social pela Universidade Complutense de Madri.

Débora Najda de Medeiros Viana é professora e Coordenadora do curso de Psicologia (UniFIP). Psicóloga (UEPB), mestre em Psicologia Social (UFPB), e aluna de doutorado da Faculdade de Ciências Médicas da Santa Casa de São Paulo (FCMSCSP).

Ana Karolyne Florencio Amorim é psicóloga (UniFIP), mestra em psicologia social (UFPB), e aluna de doutorado no Programa de Pós-Graduação em Psicologia Social (UFPB).

\section{Como citar este artigo}

Silva, F. L. (2020). Escala de Machismo Sexual: Evidências Psicométricas em Contexto Brasileiro. Avaliação Psicológica, 19(4), 420-429. http://dx.doi.org/10.15689/ap.2020.1904.15892.08 